\section{Concurrence of Torus Mandibularis with Multiple Buccal Exostoses}

\author{
Kuk Han Lee ${ }^{1}$, Jong Hun Lee ${ }^{1,2}$, Ho Jung Lee ${ }^{3}$ \\ ${ }^{1}$ Department of Plastic and Reconstructive Surgery, ${ }^{2}$ Eulji \\ Medi-Bio Research Institute, ${ }^{3}$ Department of Pathology, \\ Eulji General Hospital, Eulji University College of Medicine, \\ Seoul, Korea \\ Correspondence: Jong Hun Lee \\ Department of Plastic and Reconstructive Surgery, Eulji General Hospital, \\ Eulji University College of Medicine, 14 Hangeulbiseok-gil, Nowon-gu, \\ Seoul 139-711, Korea \\ Tel: +82-2-970-8255, Fax: +82-2-978-4772, E-mail: joaljh@eulji.ac.kr \\ This article was presented as a poster at the 69th Congress of the Korean Society of \\ Plastic and Reconstructive Surgeons on November 11-13, 2011 in Seoul, Korea. \\ No potential conflict of interest relevant to this article was reported. \\ Received: 25 Mar 2013 • Revised: 10 Apr 2013 • Accepted: 25 May 2013 \\ pISSN: 2234-6163・ elSSN: 2234-6171 \\ http://dx.doi.org/10.5999/aps.2013.40.4.466 • Arch Plast Surg 2013;40:466-468 \\ Copyright (C) 2013 The Korean Society of Plastic and Reconstructive Surgeons \\ This is an Open Access article distributed under the terms of the Creative Commons \\ Attribution Non-Commercial License (http://creativecommons.org/licenses/by-nc/3.0/) \\ which permits unrestricted non-commercial use, distribution, and reproduction in any \\ medium, provided the original work is properly cited.
}

A torus is a dense cortical bone exostosis wrapped in poorly vascularized mucosa [1]. In the oral cavity, torus mandibularis (TM) occurs above the mylohyoid line of the inner table of the body of the mandible. It mostly occurs between the last molar tooth and the lateral canine, and bilateral occurrence is well known [1-3]. Despite the fact that these bony tubercles have been under study, the apparent cause is not yet known. Buccal exostoses and tori are benign lesions and do not possess malignant potential. In these cases, they generally need no special treatment but do require periodic observation. However, if the tubercles gradually become larger or new tubercles appear, dysphagia, dysarthria, fullness of the oral cavity, or hygiene-related

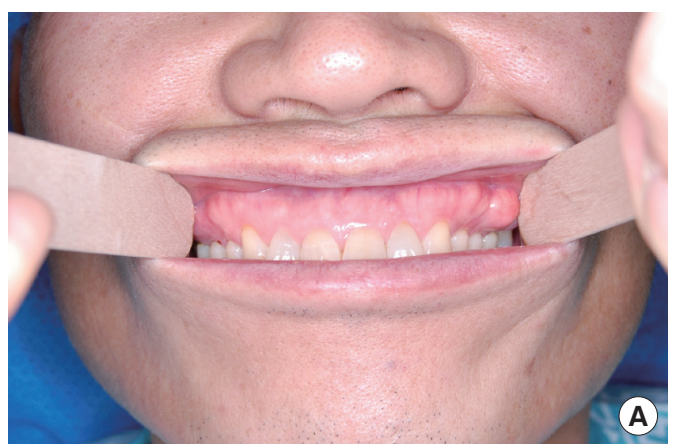

symptoms may occur in the oral cavity and cause problems when the patient is fitted with dentures. In such cases, the tubercles shoud be removed by surgery [1]. Buccal exostoses are multiple bony nodules that occur less frequently than tori. A statistical study on the incidence of tori has been published, but cases of the treatment and prognosis of patients with a concurrence of tori and multiple buccal exostosis are rarer [3]. In addition, most bony exostoses are found incidentally during dental care by a dentist because they are generally small and do not have symptoms, so plastic surgeons rarely experience them.

Thus, we present the clinical features of symptomatic tori mandibularis and multiple buccal exostoses based on our experience and treatment and survey some rare cases in the literature from the field of plastic surgery.

A 50-year-old man had a slowly growing intraoral mass that had appeared about 5 years earlier from the bone that arose from the alveolar process of the maxilla and mouth floor of the mandible. Though there was no discomfort in the early period, the tubercles were gradually becoming larger, and it held food remnants after meals and produced an intraoral foreign body sensation. In his family history, his younger sister had experienced a mandibular protuberance that was similar to the patient's lesion, but she had not visited the hospital for diagnosis or treatment. In the physical examination, multiple buccal exostoses approximately 0.5 to $1.0 \mathrm{~cm}$ in size were observed in the alveolar portions of the maxilla and both sides of the lingual aspect of the mandible, while TM were observed, about $2.5 \mathrm{~cm} \times 1 \mathrm{~cm}$ in size, on the canine to premolar region. These bony tubercles were nodular in shape and had a thin mucous membrane, but ulcers or other lesions were not observed (Fig. 1). Facial computed tomography (CT) showed

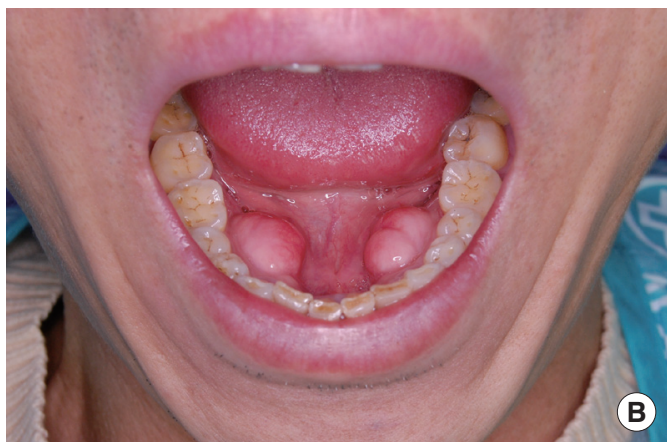

Fig. 1.

Preoperative patient findings. (A) Multiple buccal exostoses on the alveolar process of the maxilla and (B) tori madibularis. 
multiple irregularly growing hyperostoses, predominantly in the left maxilla, and symmetrically growing hyperostoses in the body of the mandible and the inner cortex. In the preoperative blood tests, the serum calcium, 25-(OH) Vitamin D, and 1.25- $(\mathrm{OH})_{2}$ Vitamin $\mathrm{D}_{3}$ were within normal limits and specific abnormal findings were not observed in other tests.

Under general anesthesia, using the appropriate osteotome at the surgical site, multiple exostoses and TM were exposed and removed. The histological appearance of the tori and buccal exostoses showed dense bony tissue, with the presence of lacunae and normal osteocytes (Fig. 2).

In most studies, it has been noted that TM occurs predominantly bilaterally [3], and it also occurred bilaterally in this case. TM usually occurs alone, but it sometimes presents with torus palatinus (TP) or buccal exostoses [3]. According to Jainkittivong and Langlais [2], the concurrence of tori and buccal exostoses is common with TM rather than TP. This is based on the fact that tori and exostoses may share the same causative factors [2]. The reported prevalence of exostoses has varied depending on distinctions among individual papers such as the regions examined and the type of research. The prevalence appears to be from $0.09 \%$ at minimum to $56 \%$ at maximum [4]. According to a previous study, those aged from 20 to 40 are known to be the age group most affected by TM [1]. The difference between the sexes in the existing studies is discordant, but it is generally known that TP is more common in females and TM in males [1,2].

The absolute cause of tori and exostoses is not yet known. The genetic factorial theory is acknowledged by most researchers, but the limits of its application are quite controversial [1]. The other potential factors worth noting are eating habits, vitamin deficiency,

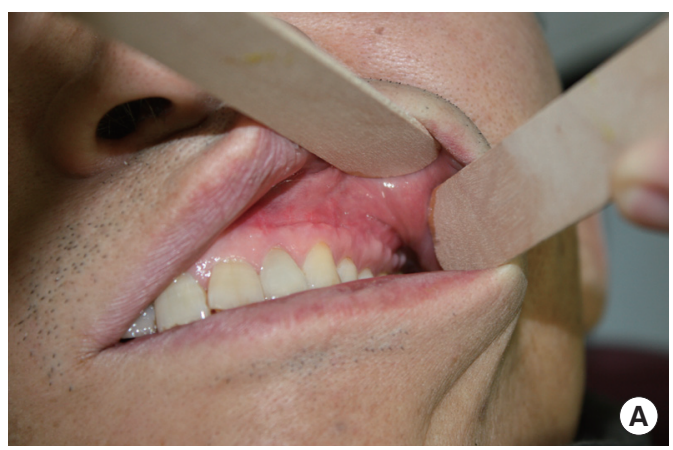

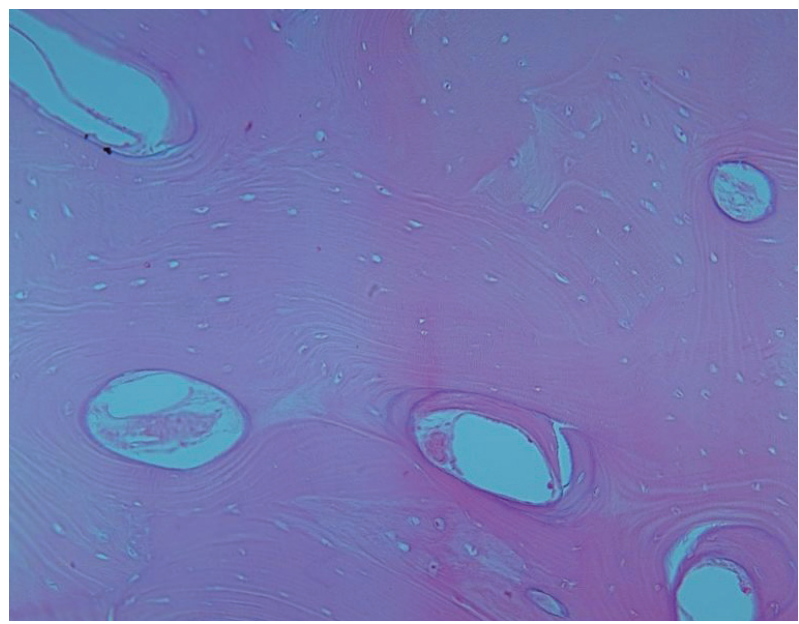

Fig. 2 .

Histological appearance. Tori mandibularis and buccal exostoses show dense bony tissue, the presence of lacunae, and normal osteocytes ( $\left.H \& E_{1} \times 400\right)$.

and excessive intake of calcium [1]. Furthermore, there are a few theories on masticatory hyperfunction or multiple environmental influences $[1-3,5]$.

The chief complaint of the patient in this case was TM with multiple buccal exostoses, and TP could not be observed. Radiologic evaluation can be done for the diagnosis of torus and exostoses, and a radiodense image can be seen by X-ray, but performing an X-ray is not useful [1]. CT is sometimes utilized for diagnosis and establishment of a treatment plan. Tori or buccal exostoses are asymptomatic, so they cannot be easily recognized. Other than that, disturbances of phonation, limitation of masticatory mechanics, sensitivity due to the thin mucosa layer, traumatic inflammation, and ulcer of a traumatic origin are the indications for extirpation $[1,4]$. In addition to that, it has been reported that TM may cause sleep apnea or difficulty of intubation under general anesthesia. Although

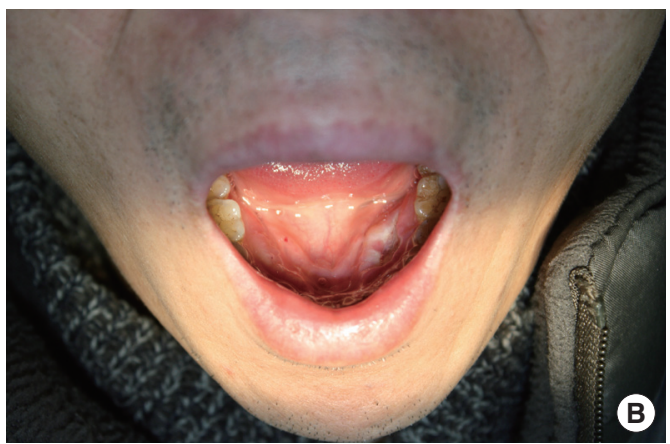

Fig. 3.

Postoperative patient findings. (A) Multiple buccal exostoses on the alveolar process of the maxilla were removed.

(B) Tori madibularis were removed, and the wound dehiscence of the left incisional site was improved. 
there is no clear criteria for classification by size, classification into groups of $2 \mathrm{~mm}, 2-4 \mathrm{~mm}$, and above $4 \mathrm{~mm}$, or $3 \mathrm{~mm}, 3-6 \mathrm{~mm}$, and above $6 \mathrm{~mm}$ is common. $2 \mathrm{~mm}$ and $3 \mathrm{~mm}$ have been found to be common sizes from these studies [1]. There is no absolute size of tori that indicates surgery, but the associated symptoms of growing tori can be an indication. The possible complications after surgery include hematoma, edema, wound dehiscence, and infection [1]. Our patient also had a thinned mucosal layer, so it was excorticated with care before closure, but wound dehiscence was observed after 6 days, so wound revision was conducted. After the revisional operation, the wound healed with no more problems (Fig. 3). This must be considered before planning surgery, and it is important to minimize morbidity on the lesion.

\section{References}

1. Garcia-Garcia AS, Martinez-Gonzalez JM, GomezFont $\mathrm{R}$, et al. Current status of the torus palatinus and torus mandibularis. Med Oral Patol Oral Cir Bucal 2010;15:e353-60.

2. Jainkittivong A, Langlais RP. Buccal and palatal exostoses: prevalence and concurrence with tori. Oral Surg Oral Med Oral Pathol Oral Radiol Endod 2000;90:48-53.

3. Antoniades DZ, Belazi M, Papanayiotou P. Concurrence of torus palatinus with palatal and buccal exostoses: case report and review of the literature. Oral Surg Oral Med Oral Pathol Oral Radiol Endod 1998;85:552-7.

4. Choi Y, Park H, Lee JS, et al. Prevalence and anatomic topography of mandibular tori: computed tomographic analysis. J Oral Maxillofac Surg 2012;70:1286-91.

5. Jainkittivong A, Apinhasmit W, Swasdison S. Prevalence and clinical characteristics of oral tori in 1,520 Chulalongkorn University Dental School patients. Surg Radiol Anat 2007;29:125-31.

Fig. 1. A right earlobe keloid; sessile-type single nodular pattern based on Chang-Park classification (type II).

\section{Earrings Embedded within Earlobe Keloids}

Ji Hae Park ${ }^{1}$, Tae Hwan Park ${ }^{2,3}$, Choong Hyun Chang

${ }^{1}$ Department of Plastic and Reconstructive Surgery, Kangbuk Samsung Hospital, Sungkyunkwan University School of Medicine, Seoul, Korea; ${ }^{2}$ Keloid Research Foundation, New York, USA;

${ }^{3}$ Deokjeok Health Care Center, Incheon, Korea

Correspondence: Choong Hyun Chang

Department of Plastic and Reconstructive Surgery, Kangbuk Samsung Hospital, Sungkyunkwan University School of Medicine, 29 Saemunan-ro, Jongno-gu, Seoul 110-746, Korea

Tel: +82-2-2001-2178, Fax: +82-2- 2001-2177, E-mail: eppeen@hanmail.net

No potential conflict of interest relevant to this article was reported.

Received: 12 Apr 2013• Revised: 28 May 2013• Accepted: 8 Jun 2013 pISSN: 2234-6163 • elSSN: 2234-6171

http://dx.doi.org/10.5999/aps.2013.40.4.468 • Arch Plast Surg 2013;40:468-469

Copyright (C) 2013 The Korean Society of Plastic and Reconstructive Surgeons

This is an Open Access article distributed under the terms of the Creative Commons Attribution Non-Commercial License (http://creativecommons.org/licenses/by-nc/3.0) medium, provided the original work is properly cited.

Keloids are a proliferative ailment of fibrous tissue secondary to dysregulation in various wound healing processes [1]. The diverse phenotypes and multitude of factors that trigger keloid formation have led us to propose "keloid disorder (KD)" as the identifying name for this condition and the term "keloid" to be reserved for referring to each individual skin lesion that patients have. Although benign, $\mathrm{KD}$ can cause aesthetic and functional problems, all of which pose a

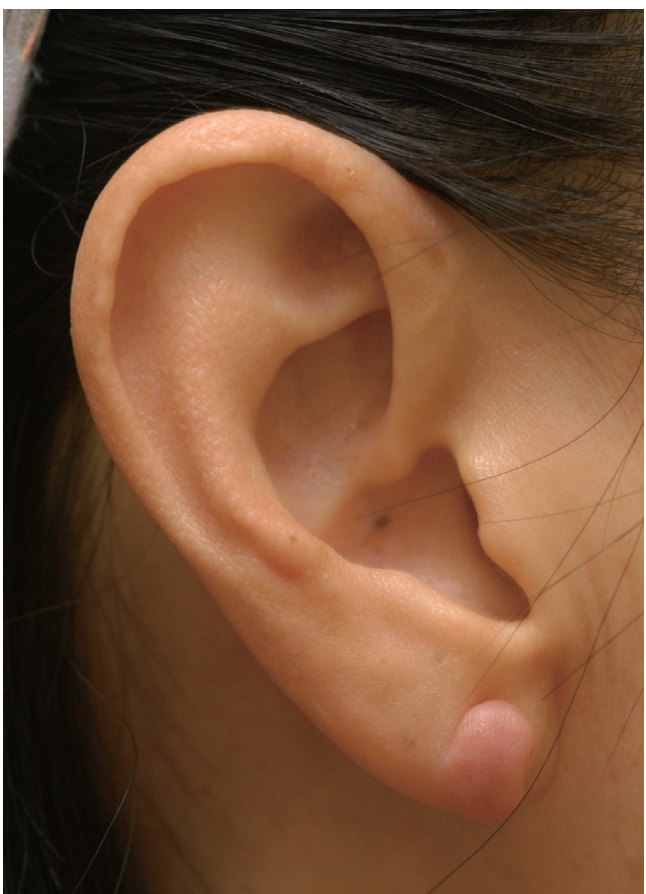

